\title{
Towards a coherent terminology of gout
}

\author{
Peter A Simkin
}

Do you think the present terminology of the various crystal deposition states and their related clinical syndromes:

A is not broken and does not need to be fixed

$B$ needs some minor adjustment

C should be made more systematic in a comprehensive revision?

How would you answer this question? When it was included in a questionnaire answered by a small group of rheumatologists with a demonstrated interest in gout, four chose A, five opted for $B$, and 17 favoured $C$. Individual comments ranged from 'I am not confused' by the existing language to 'the present terminology . . . sucks'. Overall, however, $65 \%$ of the respondents felt that real problems exist and are sufficiently severe to justify a systematic revision. ${ }^{\star}$ In this commentary I will discuss what I believe to be the principal problems and propose a simple solution.

\section{Problem 1: 'Gout' means different things to different people}

No term in rheumatology is more timehonoured than this classic diagnosis but rheumatologists apply it within quite different paradigms. To some, gout is a type of arthritis; to others, it is a systemic metabolic disease; to others, a crystal deposition state; and to still others, it is some combination of the above. There are interesting historical reasons for these differing concepts but those need not be reviewed here. Many would agree, however, that if the term is to remain useful it should be redefined so that all of us will use it consistently.

\section{Problem 2: The various pathological crystals are called by different names}

For the most part, this concern applies not to the full name of the crystal but to the way that the name is abbreviated in clinical reports. Monosodium urate monohydrate, for instance, is a clear but awkward term that is more often

Department of Medicine, RG-28, University of Washington, Seattle, WA 98195, USA P A Simkin

Correspondence to: Dr Simkin.

*The following doctors kindly returned the completed questionnaires on the terminology of gout. This listing of their names does not imply that they agree with the proposals of this
commentary. Carlos Agudelo, Anders Bjelle, Juan Canoso, John Decker, Paul Dieppe, Michael Doherty, Adel Fam, Eric John Decker, Paul Dieppe, Michael Doherty, Adel Fam, Eric
Gall, Robert Gatter, Paul Halverson, L A Healey, George Ho, James Klinenberg, Edward Lally, Stephen Malawista, Daniel J James Klinenberg, Edward Lally, Stephen Malawista, Daniel J Lawrence Ryan, Ralph Schumacher, J Edwin Seegmiller, Peter Abraham Weinberger, Robert Wortmann. Robert Terkeltaub, shortened to urate, MSU, or sometimes to the inaccurate, but traditional, uric acid. The problem is more severe for basic calcium phosphate crystals, which are variously referred to as apatite, hydroxyapatite, BCP, or the full, but cumbersome, names of the specific subtypes. Effective communication would be best served if students of pathological crystals adopted a common terminology for them.

\section{Problem 3: The various names for clinical presentations of crystal deposition} problems fail to reflect their commonalty As there are no accepted guidelines, each author is free to use the name that he or she finds most appealing. In practice, this responsibility has proved to be a heavy one and authors have distributed the burden by using several different but equivalent terms. For instance, when calcium oxalate crystals were first recognised as a cause of arthritis, the first four reports in the English language used a total of 19 essentially synonymous terms. ${ }^{1}$ Such redundancy burdens our students and colleagues, interferes with computerised retrieval of information, and provides no nosological guidance to those who recognise new species of pathological crystals. Agreement on a systematic nomenclature would clear away these unnecessary barriers to communication.

These three problems reflect the present diversity in the terminology of crystal deposition diseases. It should be emphasised that diversity in the field does not imply confusion of individuals. Those who write about this area are generally clear in their thinking and consistent in their usage. The problems arise because these usages differ. If a systematic terminology is to be adopted, each of us will have to make at least some revisions in the way that we talk and write about gout. I believe that the following suggestions comprise a workable terminology that would require the fewest changes overall.

\section{Proposal 1} articular tissues should be referred to by single, simple terms: urate, pyrophosphate, apatite, etc. For the most part these are the names of the principal anion. Additional terms that reflect hydration states and stoichiometrythat is, dihydrate, are appropriate and 
Proposed terminology of 'crystal deposition diseases'

\begin{tabular}{ll}
\hline Pathosis & Manifestation \\
\hline (Urate) $^{\star}$ gout & (Urate) gouty arthritis $\dagger$ \\
Pyrophosphate gout & Pyrophosphate (gouty) arthritis \\
Apatite gout & Apatite (gouty) arthritis \\
Oxalate gout & Oxalate (gouty) arthritis \\
Cholesterol gout & Cholesterol (gouty) arthritis \\
\hline
\end{tabular}

^Terms in parentheses are understood and need not be used routinely.

tArthritis is used for illustrative purposes. The same crystals might cause bursitis, periarthritis, tendonitis, etc.

necessary in studies of crystallisation but would be needed clinically only if different solid forms of the same physiological solute had correspondingly different sequelae. Conveniently, that does not appear to be true.

A major advantage of simple terms is the elimination of cryptic acronyms. When this issue was raised in the questionnaire, only two respondents favoured acronyms (such as MSU), five favoured the full crystal name (monosodium urate monohydrate), and 20 preferred the simple anion (urate). As Stephen Malawista (personal communication) pointed out, 'acronyms tend to shut out the uninitiated, like secret handshakes'. It was clear in other comments that acronyms breed resentment and are widely thought to impede communication. It would seem that any name so cumbersome that it must be encoded should be replaced by a single, simple, meaningful term.

\section{Proposal 2}

Gout should be redefined as a generic term encompassing all species of pathological crystallisation in articular tissues. This usage remains consistent with the Latin origin of 'gout', but it now applies to any crystal that 'drops' into a joint. Under this convention the proper names for the deposition diseases would be apatite gout, cholesterol gout, pyrophosphate gout, etc. As the prototype for all species in the genus, urate gout would be known simply as 'gout' with the species term 'urate' understood (table).

By designating each form of pathological crystallisation as the factor determining the name of the condition, this proposal underscores the central concept of crystal deposition disease. ${ }^{2}$ By considering each of them as a species of gout, we preserve a central place for one of the most traditional diagnoses in medicine. 'Urate deposition disease' or 'urate arthropathy' are much less satisfactory alternatives that are cumbersome and, more importantly, leave no place for the classic term 'gout'.

To some, it may seem inappropriate to apply 'gout' to clinical presentations apart from those of urate crystals. The idea, however, is certainly not new. The term 'kalkgicht' has long been used for apatite deposition disease in German publications and its English translation 'calcium gout' appeared in many editions of Arthritis and Allied Conditions (Hollander J, personal communication). Similarly, 'goutte oxalique' has been used as a tribute to the features linking this less common form of pathological crystallisation to the classic prototype, gout. ${ }^{3}$

\section{Proposal 3}

When crystal induced inflammation occurs the process should be denoted by the name of the underlying condition and the site of the inflammation - that is, arthritis, tendonitis, etc. In routine usage certain terms can be understood for the sake of simplicity. Thus urate gouty bursitis would simply be gouty bursitis and pyrophosphate gouty arthritis would be shortened to pyrophosphate arthritis. Additional adjectives will often be necessary to indicate the specific anatomical location of the response as well as the nature of its coursethat is, acute or chronic. When the role of crystals is uncertain, as is true of the apatite crystals found in many cases of osteoarthritis and in Milwaukee shoulder, most clinicians will probably prefer to retain the present syndrome designations.

Most rheumatic 'diseases' are complex syndromes fashioned out of recurring clinical experience and empirically defined by criteria chosen to select homogeneous groups of patients. Gout has not been an exception to this pattern, and over the years our concepts have broadened and evolved to incorporate more and more aspects of the disease. The most fundamental of these changes, however, has been the acceptance of crystal deposition as the central aspect of this condition.

There is an old saying to the effect that if you don't know where you are going you may not know when you get there. Where we are going in rheumatology is towards a logical understanding of each condition that afflicts our patients. Such understanding requires identification of central pathological factors whose causes and effects can be systematically examined. We do not have such factors for most of the syndromes we encounter, but we do for gout. There, pathological crystals of urate or pyrophosphate, for instance, are as the fundamental to their clinical sequela as the staphylococcus and gonococcus are to septic arthritis. This concept has developed and found wide acceptance over more than 30 years. It now seems appropriate to mark this acceptance by discarding the many and various names that are applied to idiopathic, crystal related syndromes and replacing them with a new terminology that is more consistent, clear, and simple but still pays homage to tradition. This commentary proposes such a system and invites the constructive responses of those who see room for improvement in the current terminology of gout.

I thank Michelle Rickard for her help in the preparation of this manuscript and the many colleagues whose comments and criticisms have helped me to formulate these concepts. Supported in part by a grant from the Upjohn Co.

1 Simkin P A. Articulate oxalate crystals and the taxonomy of gout. $\mathcal{F} A M A$ 1988; 260: 1285-6.

McCarty D J. On the crystal deposition diseases. Diseasea-Month 1970; 16: 1-29.

3 Loeper M. Rheumatisme chronique et oxalemie. Nutr Tome II Ann Clin Biol Ther 1932; 151-60. 